\title{
DESCENDINGLY INCOMPLETE ULTRAFILTERS
}

\author{
BY \\ C. C. $\mathrm{CHANG}\left({ }^{(1)}\right.$
}

Introduction. It is well known that every nonprincipal ultrafilter $D$ on a set $I$ is $\omega$-incomplete, provided the cardinal of the set $I$ is not exceedingly large. (We refer the reader to the comprehensive paper by Keisler and Tarski [5] for all relevant information and further references.) Since ultrafilters are closed under finite intersections of its members, it is easy to see that $D$ being $\omega$-incomplete is equivalent to saying that there exists a $\omega$-termed sequence

$$
X_{0} \supset X_{1} \supset \ldots \supset X_{n} \supset \cdots
$$

of elements of $D$ such that

$$
\bigcap_{n \in \omega} X_{n}=0
$$

In this note we give some partial answers to the following question: What are the cardinals $\alpha$ and ultrafilters $D$ such that $D$ will have a descending $\alpha$-termed sequence

$$
X_{0} \supset \cdots \supset X_{n} \supset \cdots, \quad \eta<\alpha,
$$

of elements of $D$ such that

$$
\bigcap_{n<\alpha} X_{n}=0 ?
$$

We say that the ultrafilter $D$ has an $\alpha$-descending sequence if it has a descending $\alpha$ termed sequence of elements as above; in which case, we also say that $D$ is descendingly $\alpha$-incomplete. The question raised above is not entirely trivial, because simply knowing that $D$ has a subset $E$ of cardinality $\alpha$ such that $\bigcap E=0$ will not automatically produce a (well-ordered) descending $\alpha$-termed sequence of elements of $D$ with intersection 0 . As examples, let us consider some simple cases when $\alpha=\omega_{1}$. It is clear that no ultrafilter $D$ on a countable set $I$ has an $\omega_{1}$-descending sequence. It is equally clear that an ultrafilter $D$ on a set $I$ with cardinality $\omega_{1}$ has an $\omega_{1}$-descending sequence if and only if every element of $D$ has power $\omega_{1}$, i.e., $D$ is uniform on $I$. Now, let us consider a nonprincipal ultrafilter $D$ on a set $I$ with cardinality $\omega_{2}$. Let $\beta$ be the smallest cardinal for which there exists a set in $D$ of that power. If $\beta<\omega_{2}$, then we have already reduced the problem to the two preceding cases, that is:

$$
\text { if } \beta=\omega \text {, then } D \text { has no } \omega_{1} \text {-descending sequence, }
$$

Received by the editors February 21, 1966.

${ }^{(1)}$ The research and preparation of this paper were partly supported by an NSF research grant. These results were announced in Chang [1]. 
and

$$
\text { if } \beta=\omega_{1} \text {, then } D \text { has an } \omega_{1} \text {-descending sequence. }
$$

Suppose $\beta=\omega_{2}$, i.e., $D$ is uniform on $I$. Then it follows as a consequence of our result Theorem 1.1 that, assuming $2^{\omega_{1}}=\omega_{2}, D$ has an $\omega_{1}$-descending sequence. This special result for arbitrary uniform ultrafilters $D$ on a set $I$ of cardinality $\omega_{2}$ is apparently new. Previously, the result was known only for certain uniform ultrafilters $D$. Generalizations of the ideas involved in this proof have led to the writing of this paper.

The original interest in this problem was motivated by studies in the cardinalities of ultraproducts of models (see, for example, Keisler [3]). In fact, the special result quoted above settled a conjecture of Keisler (private communication). We shall show in $\$ 2$ that our results have applications to ultraproducts and model theory. Theorem 2.2 is a result of Keisler and is reproduced here with his permission.

1. Set-theoretical results. We let $\alpha, \beta, \gamma$ range over cardinals, $\eta, \xi, \zeta$ range over ordinals, and $m, n$ over finite ordinals. $\alpha^{+}$denotes the cardinal successor of $\alpha$, and $\operatorname{cf}(\alpha)$ the cofinality of $\alpha .|X|$ denotes the cardinal of the set $X$. In what follows we shall prove two principal results. They are:

THEOREM 1.1. If $\alpha$ is a regular infinite cardinal such that $2^{\alpha}=\alpha^{+}$, and if $D$ is an ultrafilter having an $\alpha^{+}$-descending sequence; then $D$ has an $\alpha$-descending sequence.

THEOREM 1.2. (Assume the generalized continuum hypothesis.) If $\alpha$ is a singular cardinal and $D$ is an ultrafilter having an $\alpha^{+}$-descending sequence, then either

(i) $D$ has a $\mathrm{cf}(\alpha)$-descending sequence, or else

(ii) there exists a cardinal $\beta<\alpha$ such that for every regular cardinal $\gamma, \beta \leqq \gamma<\alpha$, $D$ has a $\gamma$-descending sequence.

We shall give the proof of Theorem 1.1 in detail and the proof of Theorem 1.2 in outline.

In all of the following lemmas let $\alpha$ be an infinite regular cardinal. Given two functions $f, g \in \alpha^{\alpha}$, we write $f \leqq g$ (or $g \geqq f$ ) if

$$
|\{\eta<\alpha: f(\eta) \leqq g(\eta)\}|=\alpha,
$$

and we write $f<g$ (or $g>f$ ) if $g$ 支, i.e.,

$$
|\{\eta<\alpha: f(\eta) \geqq g(\eta)\}|<\alpha .
$$

Clearly, $f<g$ implies $f \leqq g$.

Lemma 1.1. Let $X \subset \alpha^{\alpha}$ with $|X| \leqq \alpha$. Then there exists a function $f \in \alpha^{\alpha}$ such that

$$
f>g \text { for all } g \in X \text {. }
$$


Proof. We first well order $X$ so that

$$
X=\left\{g_{\eta}: \eta<\alpha\right\}
$$

We define the function $f \in \alpha^{\alpha}$ as follows: for each $\eta<\alpha$,

$$
f(\eta) \text { is the ordinal successor of } \bigcup_{\xi \leqq \eta} g_{\xi}(\eta) \text {. }
$$

Notice that since $\alpha$ is regular, the ordinal $\bigcup_{\xi \leqq \eta} g_{\xi}(\eta)$ is less than $\alpha$, so $f(\eta)$ is also less than $\alpha$ and $f \in \alpha^{\alpha}$. Now, given any $g \in X$, say $g=g_{\xi}$ for some $\xi<\alpha$, then for every $\eta \geqq \xi$,

$$
f(\eta)=\left[\bigcup_{\xi \leqq \eta} g_{\xi}(\eta)\right]+1>g_{\xi}(\eta)
$$

Hence $f>g$.

LEMma 1.2. Assume that $2^{\alpha}=\alpha^{+}$. There exists a subset $Y \subset \alpha^{\alpha}$ of power $\alpha^{+}$such that for every $f \in \alpha^{\alpha}$,

$$
|\{g \in Y: g \leqq f\}| \leqq \alpha .
$$

Proof. Well order the set $\alpha^{\alpha}=\left\{g_{\xi}: \xi<\alpha^{+}\right\}$. We define a sequence of functions

$$
f_{\xi} \in \alpha^{\alpha}, \quad \xi<\alpha^{+},
$$

as follows. Suppose $\xi<\alpha^{+}$and for every $\eta<\xi, f_{\eta}$ is already defined. Consider the set of functions

$$
X=\left\{f_{\eta}: \eta<\xi\right\} \cup\left\{g_{\xi}\right\} .
$$

This set has power at most $\alpha$, so by Lemma 1.1 , let $f_{\xi}$ be a function in $\alpha^{\alpha}$ which is greater than all members of $X$. Now, let

$$
Y=\left\{f_{\xi}: \xi<\alpha^{+}\right\}
$$

It is clear that $|Y|=\alpha^{+}$. Suppose $f$ is an arbitrary function in $\alpha^{\alpha}$, then $f=g_{\eta}$ for some $\eta<\alpha^{+}$. For every $\xi$ such that $\eta \leqq \xi<\alpha^{+}$, we have

$$
g_{\eta}<f_{\xi}
$$

Hence,

$$
|\{g \in Y: g \leqq f\}| \leqq \alpha .
$$

The lemma is proved.

Let $Y=\left\{f_{\xi}: \xi<\alpha^{+}\right\}$be a set of functions as in Lemma 1.2. For each $t \in \alpha \cup \alpha^{\alpha}$, we shall define a descending $\alpha$-termed sequence

$$
Y_{\xi}^{t}, \xi<\alpha,
$$

of subsets of $\alpha^{+}$as follows. Suppose first that $t \in \alpha$. For each $\xi<\alpha$, let

$$
Y_{\xi}^{t}=\left\{\eta<\alpha^{+}: \xi<f_{\eta}(t)\right\} .
$$


Notice that if $\xi \leqq \zeta<\alpha$, then

$$
\alpha^{+} \supset Y_{\xi}^{t} \supset Y_{\zeta}^{t}
$$

and, furthermore,

$$
\bigcap_{\xi<\alpha} Y_{\xi}^{t}=0
$$

Next suppose $t \in \alpha^{\alpha}$. For each $\xi<\alpha$, let

$$
Y_{\xi}^{t}=\left\{\eta<\alpha^{+}: \text {there exists a } \zeta \geqq \xi \text { such that } f_{\eta}(\zeta) \leqq t(\zeta)\right\} \text {. }
$$

Again, if $\xi \leqq \zeta<\alpha$, then

$$
\alpha^{+} \supset Y_{\xi}^{t} \supset Y_{\zeta}^{t}
$$

Intuitively, if $t \in \alpha$, then $Y_{\xi}^{t}$ is the set of all indices $\eta$ of functions $f_{\eta}$ in $Y$ such that the value of $f_{\eta}$ at $t$ is greater than $\xi$. If $t \in \alpha^{\alpha}$, then $Y_{\xi}^{t}$ is the set of all indices $\eta$ of functions $f_{\eta}$ in $Y$ such that at some future point $\zeta \geqq \xi$, the value of $f_{\eta}$ at $\zeta$ is at most the value of $t$ at $\zeta$. Thus, for such a $t \in \alpha^{\alpha}$,

implies that

$$
\eta \in \bigcap_{\xi<\alpha} Y_{\xi}^{t}
$$

there are at least $\alpha$ places $\zeta$ where $f_{\eta}(\zeta) \leqq t(\zeta)$.

This means that $f_{n} \leqq t$. But by our construction of $Y$, only at most $\alpha$ functions $f_{n}$ in $Y$ can satisfy the above, so we have

$$
\left|\bigcap_{\xi<\alpha} Y_{\xi}^{t}\right| \leqq \alpha .
$$

To summarize, we have that (1) holds if $t \in \alpha$, and (2) holds if $t \in \alpha^{\alpha}$.

LEMMA 1.3. Let $E$ be any family of nonempty subsets of $\alpha^{+}$closed under finite intersection. Then there exists a $t \in \alpha \cup \alpha^{\alpha}$ such that

$$
\left(\alpha^{+}-Y_{\xi}^{t}\right) \notin E \quad \text { for all } \xi<\alpha .
$$

Proof. Suppose to the contrary that

$$
\text { for every } t \in \alpha \cup \alpha^{\alpha} \text {, there is a } \xi<\alpha \text { such that }\left(\alpha^{+}-Y_{\xi}^{t}\right) \in E \text {. }
$$

Then, as $t$ ranges in $\alpha$, we can define a function $f \in \alpha^{\alpha}$ such that for every $\xi<\alpha$,

$$
\left(\alpha^{+}-Y_{f(\xi)}^{\xi}\right) \in E \text {. }
$$

Now, consider the sequence $Y_{\xi}^{f}, \xi<\alpha$. By our assumption (3), there is a $\zeta<\alpha$ such that

$$
\left(\alpha^{+}-Y_{\zeta}^{f}\right) \in E
$$

Now,

$$
\left(\alpha^{+}-Y_{f(\zeta)}^{\zeta}\right)=\left\{\eta<\alpha^{+}: f_{\eta}(\zeta) \leqq f(\zeta)\right\}
$$


and

$$
\left(\alpha^{+}-Y_{\zeta}^{f}\right)=\left\{\eta<\alpha^{+}: \text {for all } \xi \geqq \zeta, f_{\eta}(\xi)>f(\xi)\right\} .
$$

This means that

$$
\left(\alpha^{+}-Y_{f(\zeta)}^{f}\right) \cap\left(\alpha^{+}-Y_{\zeta}^{f}\right)=0 \in E
$$

which is a contradiction.

LEMma 1.4. Suppose that $2^{\alpha}=\alpha^{+}$and $D$ is any uniform ultrafilter on the set $\alpha^{+}$. Then $D$ has an $\alpha$-descending sequence.

Proof. Under the assumption that $2^{\alpha}=\alpha^{+}$, we construct the set $Y$ as in Lemma 1.2, and the sequences $Y_{\xi}^{t}, \xi<\alpha$, for each $t \in \alpha \cup \alpha^{\alpha}$, satisfying all of the preceding. By Lemma 1.3, we see that there exists a $t \in \alpha \cup \alpha^{\alpha}$ such that

$$
Y_{\xi}^{t} \in D, \quad \text { for all } \xi<\alpha .
$$

If $t \in \alpha$, then since, by (1), $\bigcap_{\xi<\alpha} Y_{\xi}^{t}=0, D$ has an $\alpha$-descending sequence. If $t \in \alpha^{\alpha}$, then by (2), we have

$$
\left|\bigcap_{\xi<\alpha} Y_{\xi}^{t}\right| \leqq \alpha .
$$

So the intersection is not a member of $D$. From this we see easily that the new sequence

$$
Z_{\xi}=Y_{\xi}^{t}-\left[\bigcap_{\xi<\alpha} Y_{\xi}^{t}\right]
$$

is an $\alpha$-descending sequence of elements of $D$ such that

$$
\bigcap_{\xi<\alpha} Z_{\xi}=0 \text {. }
$$

The lemma is proved.

Proof of Theorem 1.1. Let $D$ be any ultrafilter having an $\alpha^{+}$-descending sequence:

$$
Y_{0} \supset \cdots \supset Y_{\xi} \supset \cdots, \quad \xi<\alpha^{+} .
$$

For each $\xi<\alpha^{+}$, let $Z_{\xi}=\left[\bigcap_{n<\xi} Y_{\eta}\right]-Y_{\xi}$. We now define an ultrafilter $E$ on the set $\alpha^{+}$as follows: given $X \subset \alpha^{+}$, we say that

$$
X \in E \text { if and only if } \bigcup_{\xi \in X} Z_{\xi} \in D \text {. }
$$

We may verify easily that $E$ is an ultrafilter on $\alpha^{+}$. Let $X$ be any subset of $\alpha^{+}$such that $|X|<\alpha^{+}$. Then there is an $\eta<\alpha^{+}$such that

$$
\xi<\eta \quad \text { for every } \xi \in X \text {. }
$$

Hence,

$$
Y_{\eta} \cap\left[\bigcup_{\xi \in X} Z_{\xi}\right]=0
$$


and this means that $X \notin E$. So $E$ is uniform on $\alpha^{+}$. By Lemma 1.4, $E$ has an $\alpha-$ descending sequence $X_{\xi}, \xi<\alpha$, such that $\bigcap_{\xi<\alpha} X_{\xi}=0$. From this it easily follows that $D$ must also have an $\alpha$-descending sequence.

Outline of proof of Theorem 1.2. (Assume GCH.) Suppose $\alpha$ is a singular cardinal and suppose that $D$ has an $\alpha^{+}$-descending sequence. Without loss of generality (see above proof) we can assume that $D$ is a uniform ultrafilter on the set $\alpha^{+}$. Suppose that (ii) fails to hold, i.e., for every cardinal $\beta<\alpha$, there is a regular cardinal $\gamma$ such that $\beta \leqq \gamma<\alpha$ and $D$ does not have a $\gamma$-descending sequence. Let us select an increasing $\operatorname{cf}(\alpha)$-termed sequence of such cardinals, say, $\gamma_{\xi}, \xi<\operatorname{cf}(\alpha)$, so that

$$
\xi<\gamma_{\xi} \quad \text { for each } \xi<\operatorname{cf}(\alpha)
$$

and

$$
\sum_{\xi<\alpha} \gamma_{\xi}=\alpha
$$

Consider the cartesian product

$$
p=\prod_{\xi<\mathrm{cr}(\alpha)} \gamma_{\xi}
$$

We see that $|p|=\alpha^{+}$. We now repeat Lemmas 1.1-1.4 with obvious modifications, for instance, replacing the set $\alpha^{\alpha}$ by $p$ and the cardihal $\alpha$ by $\operatorname{cf}(\alpha)$. Since we have assumed that $D$ has no $\gamma_{\xi}$-descending sequences, it will follow that some $\operatorname{cf}(\alpha)$ descending sequence of the type $Y_{\xi}^{t}, \xi<c f(\alpha)$, with $t \in p$, must have all of its terms in $D$. Since, again,

$$
\left|\bigcap_{\xi<\operatorname{cr}(\alpha)} Y_{\xi}^{t}\right| \leqq \alpha,
$$

we easily see that $D$ has a $\operatorname{cf}(\alpha)$-descending sequence. Hence (i) holds.

Let us now discuss some consequences of Theorems 1.1 and 1.2 and some problems which are still open. For simplicity, in the following discussion, we shall assume the GCH whenever it is needed. From our results it follows that if $D$ has an $\omega_{\alpha+n}$-descending sequence, then $D$ has an $\omega_{\alpha}$-descending sequence, if $\omega_{\alpha}$ is regular. Thus, if $D$ has an $\omega_{n}$-descending sequence, then $D$ has an $\omega_{m}$-sequence for each $m, 0 \leqq m<n$. In particular, if $D$ is uniform on $\omega_{n}$, say, then $D$ has an $\omega_{n^{-}}$ descending sequence, so $D$ has an $\omega_{m}$-descending sequence for all $m, 0 \leqq m<n$. The case of singular cardinals is far less satisfactory. For instance, suppose $D$ has an $\omega_{\omega+1}$-descending sequence, then all we can conclude from Theorem 1.2 is that $D$ will have an $\omega$-descending sequence, a trivial result. The trouble here is that case (i) of 1.2 may hold while case (ii) fails. On the other hand, suppose $D$ has an $\omega_{\omega_{1}+1}$-descending sequence, then we can not even conclude that $D$ has an $\omega_{1}$-descending sequence, because in going down in cardinality, case (ii) of 1.2 may hold while case (i) fails. We may, of course, apply our results over and over again to those cardinals $\gamma$ for which $D$ has a $\gamma$-descending sequence, but there is 
still no assurance that $\omega_{1}$-descending sequences exist in $D$. Thus the two representative open problems are:

(I) If $D$ has an $\omega_{\omega+1}$-descending sequence then does $D$ have (a) an $\omega_{1}$-decreasing sequence? (b) an $\omega_{n}$-descending sequence for each $n$ ?

(II) If $D$ has an $\omega_{\omega_{1}+1}$-descending sequence then does $D$ have (a) an $\omega_{1}$ descending sequence? (b) an $\omega_{n}$-descending sequence for each $n$ ?

There are also two closely related problems which cannot be stated in the manner of (I) and (II). They are:

( $\left.\mathrm{I}^{\prime}\right)$ If $D$ is uniform on $\omega_{\omega}$, then must $D$ have (a) an $\omega_{1}$-descending sequence? (b) an $\omega_{n}$-descending sequence for each $n$ ?

(II') If $D$ is uniform on $\omega_{\omega_{1}}$, then must $D$ have (a) an $\omega_{2}$-descending sequence? (b) an $\omega_{n}$-descending sequence for each $n \geqq 2$ ?

Finally, one may ask to what extent is the assumption of $2^{\alpha}=\alpha^{+}$(or the GCH) necessary in our arguments.

2. Model-theoretical applications. Both of the applications we shall present in this section depend heavily on existing model-theoretical results (Theorems 2.1 and 2.2). The applications are of the following nature: knowing that $D$ has an $\alpha$-descending sequence will allow us to conclude from other results that certain things happen to $D$.

Our first application has to do with cardinalities of ultraproducts. We refer the reader to Keisler [3] for notation and other references. Suppose $D$ is a uniform ultrafilter on the set $\beta$, and $\alpha$ is an arbitrary infinite cardinal, then as in [3], we let $\alpha^{\beta} / D$ denote the cardinal of the corresponding ultrapower of $\alpha$. Let $\kappa(\alpha, \beta)$ be the smallest cardinal in the set

$$
\left\{\alpha^{\beta} / D: D \text { uniform on } \beta\right\} \text {. }
$$

Some known results concerning the function $\kappa$ are the following:

(i) $\kappa(\alpha, \alpha)>\alpha$;

(ii) $\kappa\left(\sum_{\alpha<\beta} \gamma^{\alpha}, \beta\right)=\gamma^{\beta}$ for all $\gamma \geqq 2$;

(iii) $\kappa\left(\omega_{m}, \omega_{n}\right)>\omega_{m}$ for all $m, 0 \leqq m \leqq n$;

(iv) $\kappa(\omega, \alpha)>\omega$, unless $\alpha$ is so large that there is a nonprincipal $\omega$-complete ultrafilter over $\alpha$.

The function $\kappa$ is not yet very well understood. For instance, it is suspected that

$$
\kappa(\beta, \alpha)>\alpha \text { for all } \beta \text { such that } \omega \leqq \beta \leqq \alpha,
$$

however, we have no proof. It is not even known whether $\kappa$ is a monotonically increasing function in the second argument. Many of these questions are open with or without the assumption of the GCH. Two of the simplest unknown inequalities are the following:

$$
\begin{array}{r}
\kappa\left(\omega, \omega_{1}\right)>\omega_{1} ? \\
\kappa\left(\omega_{1}, \omega_{\omega}\right)>\omega_{1} ?
\end{array}
$$


The second inequality would be true if the answer to problem $\left(\mathrm{I}^{\prime}\right)$ is yes. By using our results in $\S 1$ and the following Theorem 2.1 , we can supply a few more values of the function $\kappa$. Recall that Theorem A, part (i), of [3] states:

If $\alpha$ is infinite and $D$ is a countably incomplete ultrafilter on $\beta$, then

$$
\alpha^{\beta} / D=\left[\alpha^{\beta} / D\right]^{\omega} .
$$

A generalization of this result is the following.

THEOREM 2.1. Suppose $\gamma$ is a cardinal such that $\alpha=\sum_{\delta<\gamma} \alpha^{\delta}$ and suppose $D$ is an ultrafilter on $\beta$ with a $\gamma$-descending sequence. Then

$$
\alpha^{\beta} / D=\left[\alpha^{\beta} / D\right]^{\gamma} .
$$

Proof. This result is clearly a direct generalization of the result $\gamma=\omega$. Its proof is also a direct analog of the corresponding proof, and should present no difficulties. We shall omit the details.

Using this simple result we see that various values of $\kappa$ can now be calculated (assuming the GCH). For example suppose that

$$
\alpha=\omega_{\omega_{1}}, \beta=\omega_{2} \text {, and } \gamma=\omega_{1},
$$

then by combining 2.1 with 1.1 , we see that

$$
\kappa\left(\omega_{\omega_{1}}, \omega_{2}\right)=\omega_{\omega_{1}+1} .
$$

Clearly, various other values of $\kappa$ can be similarly calculated. Simple though results of the kind in (v) may be, we emphasize that at the moment we do not know of any other way of establishing them. One last remark. If the answer to problem $\left(\mathrm{I}^{\prime}\right)$ is yes, then by combining 2.1 with 1.1 , we have

$$
\kappa\left(\omega_{\omega_{1}}, \omega_{\omega}\right)=\omega_{\omega_{1}+1} .
$$

The second kind of applications we have in mind have to do with elementary extensions of models

$$
\mathfrak{M}=\langle A, \leqq, \cdots\rangle
$$

for a first-order language with a binary predicate $\leqq$ which is stipulated to be a simple ordering relation. An elementary extension

$$
\mathfrak{N}=\langle B, \leqq, \cdots\rangle
$$

of $\mathfrak{M}$ is said to be an end extension if every element of $B-A$ comes after every element of $A$ in the ordering $\leqq$ on $B$. Various results are known about end extensions. For instance, every model $\mathfrak{M}$ of Peano's arithmetic has a proper end extension $\mathfrak{R}$, where $\leqq$ is the natural ordering in arithmetic. The following Theorem 2.2 describes the situation completely for a certain kind of models $\mathfrak{M}_{\alpha}$ where $\leqq$ is a well-ordering relation. It gives us all the information we need to know about elementary extensions of such models. 
Let $\alpha$ be an infinite cardinal and let $\leqq$ be the natural ordering of the ordinals in $\alpha$. We use $<$ to denote $\leqq$ but not $=$. By the complete model on $\alpha$ we mean the model

$$
\mathfrak{M}_{\alpha}=\langle\alpha, \leqq, \cdots\rangle
$$

which has among its relations and functions (listed in some definite order in the ... part of the model) every finitary relation and function on $\alpha$. Let

$$
\mathfrak{N}=\langle B, \leqq, \cdots\rangle
$$

be any elementary extension of $\mathfrak{M}_{\alpha}$. Notice that the relation $\leqq$ on $B$ may not be a well-ordering relation. Let $\beta$ be any cardinal less than or equal to $\alpha$. We say that $\mathfrak{N}$ realizes $\beta$ if there exists an element $b \in B$ such that

$$
\mathfrak{R} \models \xi<b<\beta, \quad \text { for every } \xi \in \beta .
$$

Notice that in the above $\xi$ and $\beta$ are elements of $\alpha$, hence of $\mathfrak{M}_{\alpha}$, while if such an element $b$ exists it obviously must be in $B-\alpha$.

The following interesting theorem is due to Keisler (unpublished, private communication) and is given here with his permission.

THEOREM 2.2. Let $\beta, \gamma$ be cardinals such that $\beta \leqq \gamma \leqq \alpha$ and $\gamma$ is a regular cardinal. The following are equivalent:

(i) Every elementary extension $\mathfrak{N}$ of $\mathfrak{M}_{\alpha}$ which realizes $\gamma$ also realizes $\beta$.

(ii) Every uniform ultrafilter $D$ on the set $\gamma$ has a $\beta$-descending sequence.

Proof (in outline). Assume (i). Let $D$ be a uniform ultrafilter on $\gamma$. Let $\mathfrak{R}$ be the ultrapower $\mathfrak{M}_{\alpha}^{\gamma} \mid D$, so $\mathfrak{N}$ is an elementary extension of $\mathfrak{M}_{\alpha}$. The identity function $f(\xi)=\xi$ for each $\xi<\gamma$ gives rise to an element

$$
b=\left\{g \in \alpha^{\gamma}: f \sim_{D} g\right\}
$$

in $\mathfrak{R}$ such that

$$
\mathfrak{R} \models \xi<b<\gamma, \quad \text { for all } \xi \in \gamma .
$$

Hence $\mathfrak{R}$ realizes $\gamma$. By (i), $\mathfrak{N}$ realizes $\beta$. Let $c$ be an element in $\mathfrak{N}$ such that

$$
\mathfrak{N} \vDash \xi<c<\beta, \quad \text { for all } \xi \in \beta,
$$

and let $f \in \alpha^{\gamma}$ be any representative of $c$. Then, by the definition of ultraproducts, we have, for each $\xi \in \beta$, the set

$$
Y_{\xi}=\{\eta \in \gamma: \xi<f(\eta)<\beta\} \in D .
$$

Clearly, the sequence $Y_{\xi}, \xi<\beta$, is descending and

$$
\bigcap_{\xi<\beta} Y_{\xi}=0 .
$$

So $D$ has a $\beta$-descending sequence and (ii) is proved. 
Assume (ii). Let $\mathfrak{N}$ be any elementary extension of $\mathfrak{M}_{\alpha}$ having an element $b$ such that

$$
\mathfrak{N}=\xi<b<\gamma, \quad \text { for all } \xi \in \gamma .
$$

Let $D$ be the set of all subsets $P$ of $\gamma$ such that

$$
\mathfrak{N} \models P(b) \text {. }
$$

Notice that each subset $P$ of $\gamma$ must be listed among the relations in $\mathfrak{M}_{\alpha}$, hence the above line is meaningful. We can see without difficulty, using strongly the fact that $\mathfrak{N}$ is an elementary extension of $\mathfrak{M}_{\alpha}$, that

$$
\begin{aligned}
& \text { if } P, Q \in D \text {, then } P \cap Q \in D \text {; } \\
& \text { if } P \in D \text { and } P \subset Q \subset \gamma \text {, then } Q \in D \text {; } \\
& \text { if } P \cup Q=\gamma \text {, then either } P \in D \text { or } Q \in D \text {. }
\end{aligned}
$$

Hence $D$ is an ultrafilter on $\gamma$. Suppose $P \subset \gamma$ and $|P|<\gamma$. Then because $\gamma$ is regular, there will exist an element $\eta<\gamma$ such that

$$
\mathfrak{M}_{\alpha} \models \forall x(P(x) \rightarrow x \leqq \eta) .
$$

Hence

$$
\mathfrak{R} \models \forall x(P(x) \rightarrow x \leqq \eta) .
$$

This means that not $\mathfrak{N} \models P(b)$ and $P \notin D$. So $D$ is uniform on $\gamma$. By (ii), $D$ has a $\beta$-descending sequence $Y_{\xi}, \xi<\beta$. We may assume that $Y_{0}=\gamma$. We now define a function $F$ with domain $\gamma$ and range a subset of $\beta$ as follows: For each $\eta \in \gamma$

$$
F(\eta)=\text { the least ordinal } \xi \text { such that } \eta \notin Y_{\xi} \text {. }
$$

This function $F$ is among the relations listed in $\mathfrak{M}_{\alpha}$. We can express in $\mathfrak{M}_{\alpha}$ that:

the domain of $F$ is the set $\{x: x<\gamma\}$;

the range of $F$ is a subset of $\{x: x<\beta\}$;

for each $\xi \in \beta$ if $x \in Y_{\xi}$ then $F(x)>\xi$.

Because all of the above statements are also true in $\mathfrak{N}$, we see that:

$$
F(b) \text { is defined; }
$$$$
F(b)<\beta ;
$$

and, because $b \in Y_{\xi}$ for every $\xi \in \beta$,

$$
\xi<F(b)<\beta, \quad \text { for every } \xi \in \beta .
$$

Thus $\mathfrak{N}$ realizes $\beta$. The theorem is proved.

ReMARKS ABOUT THEOREM 2.2. The proof in the direction (i) implies (ii) does not require that $\mathfrak{M}_{\alpha}$ is a complete model or that $\gamma$ is regular. The proof in the other 
direction only required that $\mathfrak{M}_{\alpha}$ be complete only with respect to the binary relations and functions on $\alpha$. Actually, in this direction of the proof one can exploit the very much similar ideas in Chang [2] and show that if $\mathfrak{R}$ realizes $\gamma$ then many different elements of $\mathfrak{R}$ can be found which realizes $\beta$.

An example of an application of 2.2 and 1.1 is the following. Suppose that $\alpha \geqq \omega_{2}$ and $2^{\omega_{1}}=\omega_{2}$. Then every elementary extension $\mathfrak{R}$ of $\mathfrak{M}_{\alpha}$ which realizes $\omega_{2}$ also realizes $\omega_{1}$.

Obviously, the solutions to some of the problems we raised in $\$ 1$ will give new results here.

In conclusion, we would like to point out that the ideas underlying Theorem 2.2 can be generalized and extended to models of set theory, see Keisler [4]. Then our results 1.1 and 1.2 have corresponding applications to the so-called natural models of set theory.

\section{REFERENCES}

1. C. C. Chang, Descendingly incomplete ultrafilters, Abstract, J. Symbolic Logic 30 (1965), 269-270.

2. - A simple proof of the Rabin-Keisler theorem, Bull. Amer. Math. Soc. 71 (1965), 642-643.

3. H. J. Keisler, On cardinalities of ultraproducts, Bull. Amer. Math. Soc. 70 (1964), 644-647.

4. —- Extending models of set theory, Abstract, J. Symbolic Logic 30 (1965), 269.

5. H. J. Keisler and A. Tarski, From accessible to inaccessible cardinals, Fund. Math. 53 (1964), 225-308.

UNIVERSITY OF CALIFORNIA,

Los ANGeles, California 